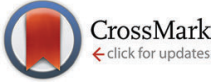

Cite this: J. Mater. Chem. B, 2016, 4, 1405

Received 27th October 2015, Accepted 20th January 2016

DOI: $10.1039 / c 5 t b 02240 a$

www.rsc.org/MaterialsB

\section{Polycaprolactone fibers with self-assembled peptide micro/nanotubes: a practical route towards enhanced mechanical strength and drug delivery applications $\dagger$}

\author{
M. S. Liberato, ${ }^{a}$ S. Kogikoski Jr., ${ }^{a}$ E. R. da Silva, ${ }^{a}$ D. R. de Araujo, ${ }^{a}$ S. Guha ${ }^{b}$ and \\ W. A. Alves*a
}

\begin{abstract}
Peptide-based scaffolds are a frontier research area in materials science with widespread impact in biomedical engineering. In this paper, we describe a hybrid material formulated through the conjugation of electrospun polycaprolactone (PCL) fibers and micro/nanotubes of L,L-diphenylalanine (FF-MNTS). Morphology and crystallinity of the composite matrices are investigated using a wide range of analytical techniques including electron microscopy, thermal analyses, X-ray diffraction and micro-tomography. Peptide assemblies are found to produce deep modifications on the microstructure of PCL fibers, impacting average diameters, crystallinity degree and porous size in the polymer network. These changes are correlated with mechanical properties of the resulting scaffolds, whose strength is found to exhibit a brittle-to-ductile transition upon increasing the amount of FF-MNTs and lead to enhanced Young's moduli of polymer fibers. The PCL/FF-MNTs composites were tested for the drug delivery application of a lipophilic drug, benzocaine. In vitro permeation studies have shown that these polymer/ peptide hybrids are able to produce a steady release of benzocaine over periods of up to $\sim 13$ hours, much higher than commercially available gel formulations. Enzymatic tests have shown a significant increment in biodegradation rates in PCL/FF-MNTs hybrids containing higher peptide amounts, which exhibited almost $100 \%$ weight loss against only $10 \%$ found in pure PCL. Our findings indicate that using PCL/FF-MNTs materials is a simple route towards achieving enhanced mechanical strength of PCL networks that have the ability to promote controlled drug delivery from a completely biodegradable matrix.
\end{abstract}

\section{Introduction}

Polymer-based skin patches for drug delivery applications should be malleable, hydrophilic and have a slow uptake of the drug in the body. ${ }^{1,2}$ However, the non-trivial balance of mechanical and thermal properties often required for attaining these characteristics represents a major challenge toward the full exploitation of these functional materials. ${ }^{1,3,4}$ Designing nanocomposites with the addition of organic or inorganic selfassembled nanostructures into polymeric matrices is a promising strategy to overcome this drawback. ${ }^{5}$ Bio-inspired self-assemblies such as L,L-diphenylalanine micro/nanotubes (FF-MNTs) are suitable for designing nanocomposite materials due to their

\footnotetext{
${ }^{a}$ Centro de Ciências Naturais e Humanas, Universidade Federal do ABC, 09210-580, Santo André, SP, Brazil. E-mail: wendel.alves@ufabc.edu.br

${ }^{b}$ Department of Physics and Astronomy, University of Missouri, Columbia, Missouri 65211, USA

$\dagger$ Electronic supplementary information (ESI) available: More data on SEM and micro-CT analyses, and mechanical properties. See DOI: 10.1039/c5tb02240a
}

excellent mechanical strength $(19 \mathrm{GPa}),{ }^{6,7}$ thermal degradation stability (up to $200{ }^{\circ} \mathrm{C}$ ), and resistance to disassembly in either organic solvents or water. ${ }^{8}$ Moreover, the aggregation process may be controlled and leads to different morphologies, which may contribute to a better dispersion and more cohesive interaction with different polymeric materials. ${ }^{9,10}$

Although several reports are available on the usage of selfassembled nanostructures for enhancing mechanical strength in nanocomposites, detailed studies on peptide-based scaffolds incorporated into polymer matrices remain relatively scarce in the literature. ${ }^{11}$ Recently, FF-MNTs were used as reinforcement agents in an epoxy matrix prepared by the solvent casting method. ${ }^{12}$ The incorporation of these structures has led to an impressive increase of shear strength, whereas thermal and elongation properties of the epoxy polymer have been found to remain preserved. ${ }^{12}$ Other studies have investigated the selfassembly of cyclic peptides into poly-D,L-lactide (PDLLA) solutions, and found that the formation of peptide microcrystals is suitable for increasing the stiffness and Young's modulus of the hybrid composites. ${ }^{13}$ In addition, fibers based on a block copolymer of 


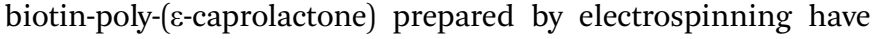
been used as templates for promoting spatial organization of biomolecules within the electrospun material. ${ }^{14}$ Interestingly, this material has shown a dynamic gradient of the arranged biomolecules reproducing gradient conditions found in biological tissues. $^{14}$

The FF-MNTs were already evaluated as potential vehicles for drug delivery using a model compound to mimic a drug. ${ }^{15}$ It has been found that FF-MNTs were able to modulate the release of the load, indicating their ability to deliver drugs at constant rates in the body. Cytotoxicity investigations revealed high cell viability up to concentrations of $5 \mathrm{mg} \mathrm{mL}^{-1}$, demonstrating the low toxicity and potential biocompatibility of FF-MNTs. On the other hand, poly- $\varepsilon$-caprolactone (PCL) is a very attractive material widely investigated for biomedical applications. ${ }^{16-19}$ Nevertheless, the appropriate designing of scaffolds for drug delivery often needs the addition of compatibilizers, which change the chemical characteristics and have an indirect effect on the mechanical characteristics, crystallinity, solubility, and degradation behaviours. Unfortunately, these changes in the finer chemistry of the matrix usually lead to a significant loss of properties suitable for drug delivery. ${ }^{19-21}$

In this work, we design an unedited peptide-based composite built from the conjugation between FF-MNTs and PCL. The peptide and the polymer were co-solubilized and subjected to electrospinning for producing fibers with controlled tensile properties. We found that the addition of FF to fibrous PCL significantly enhances the elastic modulus of the electrospun scaffolds. This approach has created stronger fibers with lengths reaching the micrometer scale and only a few hundred nanometers in diameter. Scaffolds exhibit porous morphology with diameters ranging from 360 to $570 \mathrm{~nm}$, depending on the peptide amount in the matrix. Differential scanning calorimetry (DSC) and X-ray powder diffraction (XRPD) show that the microstructure of the polymeric matrices is strongly affected by the presence of FF-MNTs, with important consequences on the elastic behaviour of the hybrid material. The potential of these scaffolds for controlled drug release is demonstrated using a lipophilic anaesthetic, benzocaine (BZC), as load. The resulting network, with interconnected pores across the polymer matrix, is suitable for drug delivery applications and exhibits good biodegradability properties. X-ray microtomography (microCT) clearly shows a reduction in porosity upon addition of FF-MNTs, which has been interpreted as an advantage for producing membranes for steady release of the drug load.

\section{Experimental section}

\subsection{Materials}

All reagents used in the experiments were of analytical purity. Chloroform, methanol and sodium azide were purchased from Synth (Brazil). The alcohol 1,1,1,3,3,3-hexafluoro-2-propanol (HFP), L,L-diphenylalanine (FF), poly-( $\varepsilon$-caprolactone) (PCL) $\left(\mathrm{M}_{\mathrm{w}}\right.$ : 70000-90000) and proteinase-K were purchased from Sigma-Aldrich (USA) and used without further purification.

\subsection{Preparation of FF-PCL composites}

Polymer fibers were obtained by electrospinning organic solutions containing FF and PCL. Lyophilized L,L-diphenylalanine (FF) powder was dissolved in 1,1,1,3,3,3-hexafluoro-2-propanol (HFP) and added to PCL solutions prepared in the chloroform/methanol mixture $(1: 3 \mathrm{v} / \mathrm{v})$. The mass percentage of polymer in the solution was kept at $8.0 \mathrm{wt} \%$, whereas the peptide/PCL ratios ranged from $2.5 \%$ to $50 \%$. Co-solubilization was attained upon continuous stirring over a period of 2 hours at room temperature. The mixtures were transferred to $15 \mathrm{~mL}$ syringes with $12 \mathrm{G}$ needles. Syringes were placed in the vertical position and dripping flow was obtained due to gravity. Square $15 \times 15 \mathrm{~cm}^{2}$ plates, covered with aluminum foils, were positioned at $15 \mathrm{~cm}$ from the needles and a voltage of $22 \mathrm{kV}$ was established for spinning fibers. The collection time was about 4 hours.

\subsection{Electron microscopy analysis}

Scanning electron microscopy images of composite membranes were obtained on a high-resolution JSM 6330F instrument (SEM-FEG), at the Center for Research in Energy and Materials (CNPEM, Campinas, Brazil). Secondary electrons were collected after back-scattering from Au-coated samples hit by electron beams with $5 \mathrm{kV}$ energy.

\subsection{X-ray powder diffraction}

Diffraction experiments were conducted at XRD1 beamline at the Brazilian National Synchrotron facility (LNLS, Campinas, Brazil). The energy of photons was set to $10 \mathrm{keV}$ and counting rates were adjusted to $1 \times 10^{7}$ photons $\mathrm{s}^{-1}$. The beam had rectangular cross-section with dimensions $2 \times 0.2 \mathrm{~mm}^{2}$, and a Mythen $1 \mathrm{~K}$ strip detector, composed of 1280 channels with 50 microns each, was used to record the data. The angular range was scanned in the interval of $10^{\circ}<20^{\circ}<30^{\circ}$, with steps of $0.005^{\circ}$. Calibration was performed using $\mathrm{Al}_{2} \mathrm{O}_{3}$ standards and instrumental broadening was estimated at $\Delta 2 \theta \sim 0.04^{\circ}$. The set-up was mounted in transmission mode and polymer membranes were conveniently placed on metallic grids.

\subsection{X-ray micro-tomography}

X-ray micro-CT measurements were performed using a Bruker SkyScan 1272 instrument. The X-ray source was operated at a voltage of $20 \mathrm{kV}$ and current of $175 \mu \mathrm{A}$. Data were recorded using a 2D X-ray detector with 16 megapixels, with the pixel size of $3.37 \mu \mathrm{m}$. Specimens with dimensions $8.5 \times 0.2 \times 0.14 \mathrm{~mm}^{3}$ were positioned along the vertical axis of a goniometer and rotated at angular steps of $0.4^{\circ}$. In this configuration, a $z$-resolution of about 350 was achieved and 450 images were acquired to cover an angular range of $180^{\circ}$. Image treatment and 3D reconstructions were performed using manufacturer's software DataViewer and CTVox. The porosity was measured using CTAn software (SkyScan, Bruker microCT), with a selected voxel coefficient.

\subsection{Mechanical analyses}

Mechanical analyses were performed using samples with dimensions $12.9 \times 6.3 \times 0.12 \mathrm{~mm}^{3}$ using a Dynamic Mechanical 
Analyzer (DMA) - TA Instruments (DMA Q800) in extension mode. Stress $\times$ strain curves were obtained using displacement rates of $700 \mu \mathrm{m} \mathrm{min}{ }^{-1}$ (ASTM D882).

\subsection{Differential scanning calorimetry}

Differential scanning calorimetry (DSC) assays were performed on TA Instruments DSC Q-series apparatus. Samples were first heated from room temperature up to $40{ }^{\circ} \mathrm{C}$, then cooled down to $-90{ }^{\circ} \mathrm{C}$. In the following, they were heated to $220{ }^{\circ} \mathrm{C}$ and cooled down to $-65{ }^{\circ} \mathrm{C}$. Data exhibited in Fig. 2 (and ESI $\dagger$ ) have been recorded during this second heating/cooling cycle. The temperature rate of $10{ }^{\circ} \mathrm{C} \mathrm{min}^{-1}$ was used throughout the experiments, which were performed in a nitrogen atmosphere.

\subsection{In vitro permeation assays}

In vitro permeation assays were performed using vertical Franz-type diffusion cells of $0.6 \mathrm{~cm}^{2}$ (Vidrotec ${ }^{\circledR}$, Porto Alegre-RS, Brazil) with artificial membranes (nitrocellulose sheets, $0.05 \mu \mathrm{m}$ pore size) impregnated with isopropyl myristate to simulate hydrophobicity of stratum corneum ${ }^{22}$ under occlusive (vapor-permeable) conditions. The donor compartment was filled with $1.0 \mathrm{~g}$ of the formulations and the receptor compartment with $5 \mathrm{mM}$ Hepes buffer containing a $0.9 \% \mathrm{NaCl}$ solution, $\mathrm{pH} 7.4$, at $32.5^{\circ} \mathrm{C}$ under constant magnetic stirring $(350 \mathrm{rpm})$. At predetermined time intervals, aliquots from the receptor compartment were analyzed using a UV-vis spectrophotometer to quantify the released drug content. Analysis was performed by using a calibration curve obtained previously $\left(y=-0.07881+0.2306 X, R^{2}=0.9989\right.$, limit of quantification $0.009 \mu \mathrm{g} \mathrm{mL}{ }^{-1}$ and limit of detection $=$ $0.053 \mu \mathrm{g} \mathrm{mL}{ }^{-1}$ ). Data were expressed as percentage or mean $\pm \mathrm{SD}$ and subjected to one-way ANOVA with post hoc Tukey-Kramer test using Graph Pad Instat (Graph Pad Software Inc., USA) or Origin 6.0 (Microcal ${ }^{\mathrm{TM}}$ Software, Inc., Northampton, MA, USA) programs. Statistical differences were defined as $p<0.05$.

\subsection{Biodegradability}

Composite membranes were weighed before incubation at $37^{\circ} \mathrm{C}$ in $0.05 \mathrm{~mol} \mathrm{~L}^{-1}$ (pH 8.6) Tris-HCl buffer solution containing $1.0 \mathrm{mg}$ of proteinase-K enzyme and $0.02 \%$ sodium azide. The buffer/enzyme solution was monitored over 25 hours. At time intervals of 2 hours, the membranes were removed from the solution, washed, vacuum dried and then weighed. The percentage of weight loss was obtained just by computing the mass loss over the initial mass using the expression $\left[\left(m_{\mathrm{after}}-m_{0}\right) / m_{0}\right] \times 100$, where $m_{\text {after }}$ is the vacuum dried weight and $m_{0}$ is the initial weight of each sample.

\section{Results and discussion}

\subsection{Morphology investigation}

Scanning electron micrographs of peptide/PCL samples, Fig. 1, show the formation of uniform fibers that are homogeneously distributed across the surface of aluminum foils used as a substrate for collection during electrospinning (additional images are provided in the ESI, $\dagger$ Fig. S1). One can observe
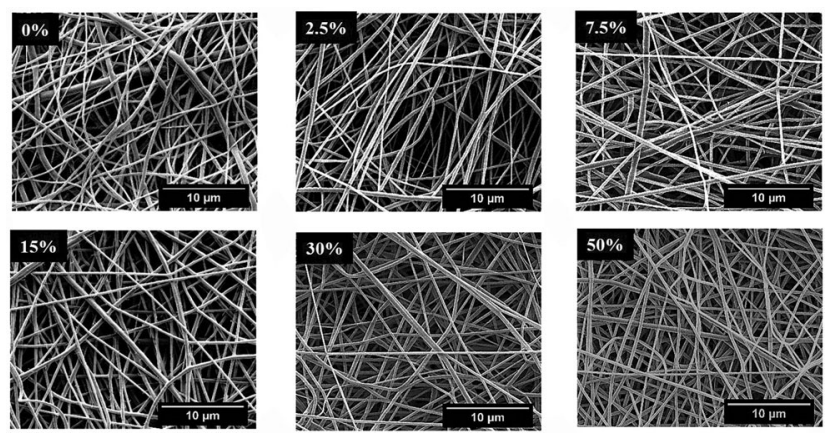

Fig. 1 Scanning electron micrographs of polymeric electrospun membranes at different FF/PCL percentages.

randomly oriented fibers, with lengths reaching the micrometer scale, and thicknesses ranging from $\sim 360 \mathrm{~nm}$ to $\sim 570 \mathrm{~nm}$, consistently forming networks with nonwoven fabric-like features. Interestingly, the general morphology of PCL fibers does not change upon addition of FF; however, their diameters are found to consistently decrease as the amount of peptide is increased in the matrix (see Table S1, ESI $\dagger$ ). These findings could be tentatively ascribed to the electrostatic attraction between the polymer and peptides, which potentially hinders the association between PCL fibrils and suppresses the growth of larger structures. Additionally, charges in the solution during jet formation may interact with the external electric field, stretching the fibers and thus reducing the diameters. ${ }^{23}$

To get a deeper insight into the role of self-assembled $\mathrm{FF}$ in the formation of composite structure, we heated the polymer networks up to $180{ }^{\circ} \mathrm{C}$, under controlled atmospheric conditions. The heating temperature was chosen to be much higher than the PCL melting point $\left(\sim 60^{\circ} \mathrm{C}\right)$, but well below the FF degradation temperature $\left(\sim 220{ }^{\circ} \mathrm{C}\right) .{ }^{8,24,25}$ Changes in sample morphology are clearly observed in comparison with the non-heat treated films (see Fig. S2, ESI $\dagger$ ). The polymer appears aggregated and forms an amorphous melted phase whereas FF-MNTs remain stable. These findings are a strong indicator that the polymer behaves as a template guide for the peptide self-assembly, and FF nanotubes remain mostly hosted within polymer fibers, forming a core-shell architecture. In addition, the systematic absence of FF-MNTs in the interstice of the polymer network supports the incorporation of peptide assemblies in the core of the fibers. Since the size of the fibers can be managed by controlling the electrospinning parameters such as the voltage, the flow rate and the peptide/ polymer ratio, this strategy has great potential for templating and synthesizing these hybrid scaffolds incorporating peptides.

\subsection{DSC and XRPD assays}

Differential scanning calorimetry (DSC) and X-ray powder diffraction (XRPD) have been performed to probe intermolecular interactions and assess how peptides affect the semi-crystalline nature of the PCL matrix. As discussed further, these data have been crucial to correlate striking changeovers on mechanical properties of the hybrid materials and the ordering state of polymer chains. In Fig. 2, thermograms of samples containing 

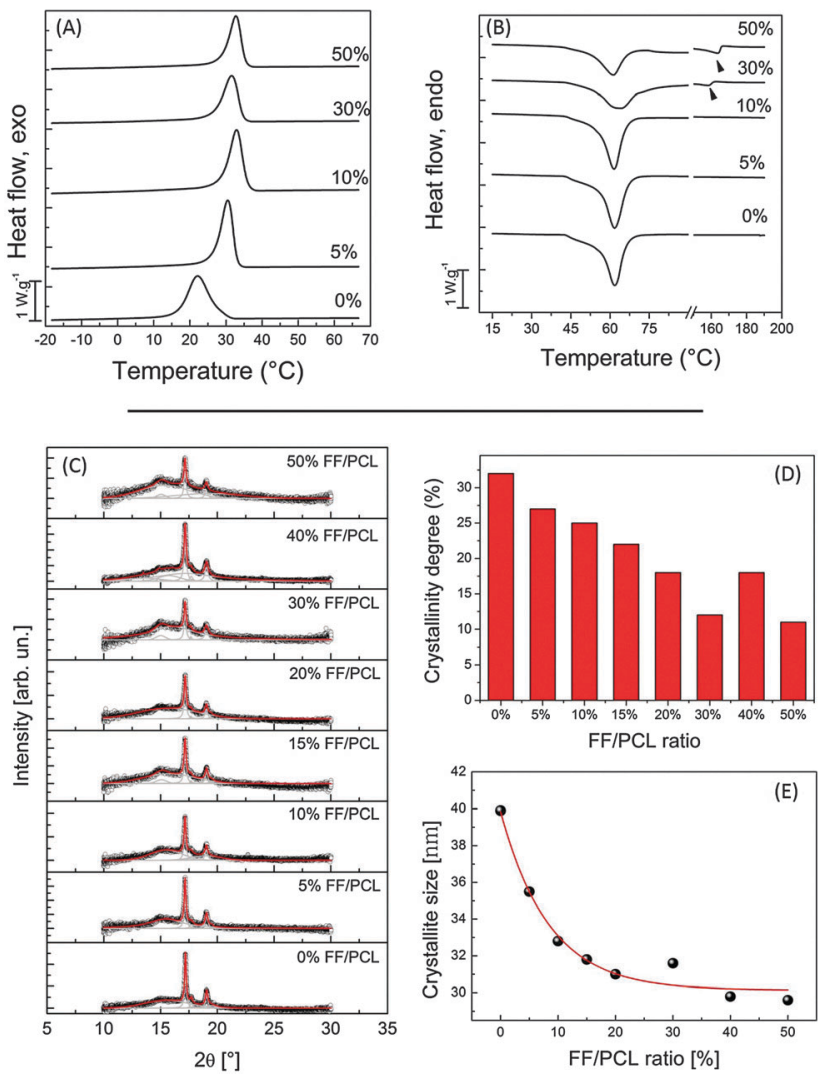

Fig. 2 Top: DSC scans of FF/PCL samples showing heat flows associated with (A) crystallization and (B) melting of PCL chains. Black arrows in (B) indicate phase transitions associated with peptide self-assemblies hosted in the interstice of the polymer matrix; bottom: (C) synchrotron X-ray powder diffraction patterns of PCL matrices containing peptides at the indicated FF/PCL percentages. Gray lines: Gaussian functions used for deconvoluting Bragg peaks and diffuse (amorphous) scattering and red lines are the summation of deconvoluted functions; (D) crystallinity degree obtained from XRPD; and (E) crystallite size calculated from the most intense Bragg peak (110), see text for details.

different FF/PCL ratios are shown. Thermal parameters arising from these assays - namely, crystallization and melting temperatures, enthalpies, entropies and crystallinity degrees - are exhibited in Table 1.

Thermograms are characterized by exothermic (Fig. 2A) and endothermic peaks (Fig. 2B), respectively, corresponding to crystallization and melting processes in the polymer phase. ${ }^{26,27}$ In samples containing higher $\mathrm{FF}$ amounts, endothermic peaks are also observed around $160{ }^{\circ} \mathrm{C}$ (pointed by black arrows in Fig. 2B), which has been ascribed to hexagonal-to-orthorhombic transitions

Table 1 Thermal parameters and crystallinity degrees arising from DSC analyses

\begin{tabular}{lllllll}
\hline $\begin{array}{l}\text { FF/PCL } \\
\text { ratio }(\%)\end{array}$ & $\begin{array}{l}T_{\mathrm{c}} \\
\left({ }^{\circ} \mathrm{C}\right)\end{array}$ & $\begin{array}{l}\Delta H_{\mathrm{c}} \\
\left(\mathrm{J} \mathrm{g}^{-1}\right)\end{array}$ & $\begin{array}{l}T_{\mathrm{m}} \\
\left({ }^{\circ} \mathrm{C}\right)\end{array}$ & $\begin{array}{l}\Delta H_{\mathrm{m}} \\
\left(\mathrm{J} \mathrm{g}^{-1}\right)\end{array}$ & $\begin{array}{l}\Delta S_{\mathrm{m}} \\
\left(\mathrm{J} \mathrm{C}^{-1} \mathrm{~g}^{-1}\right)\end{array}$ & $\begin{array}{l}X_{\mathrm{c}} \\
(\%)\end{array}$ \\
\hline 0 & 22 & 49.5 & 61 & 64.4 & 0.19 & 48 \\
5 & 31 & 45.1 & 60 & 53.3 & 0.16 & 41 \\
10 & 33 & 44.1 & 63 & 49.2 & 0.15 & 40 \\
30 & 31 & 37.5 & 62 & 39.7 & 0.12 & 38 \\
50 & 33 & 33.5 & 60 & 29.9 & 0.09 & 33
\end{tabular}

in the crystalline structure of FF-MNTs. ${ }^{28-30}$ Focusing on the thermal behaviour associated with the polymer phase, one observes that crystallization temperature is found at $T_{\mathrm{c}}=22{ }^{\circ} \mathrm{C}$ in bare PCL matrices whereas composites exhibit an impressive jump with $T_{\mathrm{c}}$ increasing up to $33{ }^{\circ} \mathrm{C}$ in FF-containing samples (see Fig. 2A). In contrast, apparently melting temperatures are not strongly affected by peptide species and remain stable around $T_{\mathrm{m}}=61.2 \pm 1.3^{\circ} \mathrm{C}$ (Fig. 2B). However, more remarkable changes in the thermal behaviour of the composites are related to crystallization/melting enthalpies. In fact, one observes a monotonic decrease in specific enthalpies associated with either exothermic or endothermic transitions (see Table 1).

Crystallization enthalpy is found to decrease from $\Delta H_{\mathrm{c}}=$ $49.5 \mathrm{~J} \mathrm{~g}^{-1}$ in bare PCL down to $\Delta H_{\mathrm{c}}=33.5 \mathrm{~J} \mathrm{~g}^{-1}$ in the $50 \%$ (FF/PCL) sample. Similarly, melting enthalpy decreases from $\Delta H_{\mathrm{m}}=64.4 \mathrm{~J} \mathrm{~g}^{-1}$ to $\Delta H_{\mathrm{m}}=29.9 \mathrm{~J} \mathrm{~g}^{-1}$. These findings indicate that the presence of FF species in the interstice of the polymer phase likely weakens the strength of inter-chain interactions, directly affecting the crystalline behaviour as suggested by the broadening of endothermic peaks. To quantify these modifications, we have used the enthalpy data listed in Table 1 to calculate the crystallinity degree in our samples using the relationship: $X_{\mathrm{c}}(\%)=\left[\Delta H_{\mathrm{m}} /\left(w \times \Delta H_{\mathrm{m}}^{\infty}\right)\right] \times 100$, where $\Delta H_{\mathrm{m}}^{\infty}$ is the heat of fusion for $100 \%$ crystalline PCL (here, assumed to be $\left.\Delta H_{\mathrm{m}}^{\infty}=135 \mathrm{~J} \mathrm{~g}^{-1}\right)^{31}$ and $w$ is the weight fraction of the polymer in the sample. The degree of crystallinity obtained from the thermal data is found to decrease from $X_{\mathrm{c}}=48 \%$, in the pure PCL formulation, to about $X_{\mathrm{c}}=33 \%$ in the sample formulated with $50 \% \mathrm{FF} / \mathrm{PCL}$. This finding is consistent with loss of ordering arising from weaker interactions between polymer chains.

Entropic contributions also play an important role in the FF/PCL composites described here. To estimate the entropy gain in different samples, we have determined heat capacity $\left(C_{\mathrm{p}}\right)$ as a function of temperature (ESI, $\dagger$ Fig. S3). Formulations containing FF clearly exhibit higher $C_{\mathrm{p}}$ values when compared to pristine PCL. Plots of $C_{\mathrm{p}} / T \times T$, integrated in the range between $0{ }^{\circ} \mathrm{C}$ and $30{ }^{\circ} \mathrm{C}$, reveal strong entropy gain and attest that higher amounts of peptide lead to higher levels of disorder in the mixtures (see ESI $\dagger$ for details). This behaviour is likely a consequence of mixing entropy which appears in the system upon addition of peptides to the formulations. Furthermore, it could be also correlated to the growth of configurational entropy of polymer chains, which is consistent with the diminution of crystallite sizes revealed by X-ray assays (see below).

Entropies of fusion, $\Delta S_{\mathrm{m}}$, also have been estimated. At the melting point, equilibrium is reached and Gibbs free energy change is null: $\Delta G_{\mathrm{m}}=\Delta H_{\mathrm{m}}-T \times \Delta S_{\mathrm{m}}=0$. To a first approximation, we have assumed $T=T_{\mathrm{m}}$ throughout the melting process and then $\Delta S_{\mathrm{m}}$ values could be calculated straightforward by using $\Delta H_{\mathrm{m}}$ and $T_{\mathrm{m}}$ data obtained previously. Estimations for $\Delta S_{\mathrm{m}}$ are listed in Table 1 and they reveal that entropy of fusion decreases upon increasing the amount of peptide in the mixture. This result is in line with higher levels of disorder found in the solid phase of $\mathrm{FF} / \mathrm{PCL}$ composites. In fact, since crystallinity is lower when FF is present in the polymer matrix, the entropy gain needed for fusion presumably should be lower. 
To further understand the structure of our peptide/polymer composites and obtain independent measurements on the crystallinity degree, we have performed synchrotron XRPD experiments. Furthermore, these assays have allowed us to quantify the crystallite size in the samples. Data from these measurements are shown in Fig. 2C, where one observes that diffractograms are dominated by intense Bragg peaks at $2 \theta \sim 17.2^{\circ}$ and $\sim 19.1^{\circ}$, accompanied by shallow shoulders at $17.7^{\circ}$ and $19.5{ }^{\circ} \mathrm{C}$. These reflections, in an increasing angular order, are attributed to the Miller indices (110), (111), (200) and (201) of an orthorhombic unit cell with $a=7.55 \AA, b=4.97 \AA$ and $c=17.27 \AA$, in close agreement with previous literature which has found that PCL chains are organized into a $P 2_{1} 2_{1} 2_{1}$ space group. ${ }^{31}$ Additionally, a broad peak centered at $2 \theta \sim 15^{\circ}(011)$ appears convoluted with the diffuse contribution of the amorphous PCL phase, which increases upon FF addition likely due to weakening of intermolecular interactions in the matrix. The crystallinity degree was obtained from XRPD data by considering the ratio between the areas under the Bragg peaks and the total scattering across the angular range (including the amorphous phase). Contributions from both Bragg peaks and diffuse scattering have been deconvoluted by adjusting Gaussian functions and the corresponding areas have been calculated (see gray lines in Fig. 2C). In Fig. 2D, we show crystallinity degrees arising from this procedure where a decrease in crystallinity is observed upon peptide addition, in agreement with the general trend found on DSC data (Table 1). In addition, we observe that crystallinity values estimated from PXRD data are lower than those estimated from DSC assays. This behaviour has also been observed elsewhere $^{31}$ and it is ascribed to the partial crystallization of the amorphous polymer during heating/cooling cycles which contribute to the increasing degree of ordering in DSC experiments. In addition, since our samples have fibrillar structures, the presence of anisotropy in our membranes potentially leads to the underestimation of crystallinity obtained from X-ray assays. Crystallite sizes have been calculated from the most intense reflection (110) by using the Scherrer equation: ${ }^{32} t=(0.9 \times \lambda) /$ $(\beta \times \cos \theta)$, where $t$ is the crystallite size, $\lambda$ is the $\mathrm{X}$-ray wavelength and $\beta$ is the full width at maximum half (FWHM) of the Bragg peak centered at angle $\theta$. Overall, one observes that the size of the crystalline domains decreases upon increasing FF in the formulation, dropping from $t \sim 35.5 \mathrm{~nm}$, in the pure PCL matrix, to $t \sim 29.5 \mathrm{~nm}$ in the formulation containing the highest amount of peptides.

In light of the structural data described above, we propose two mechanisms by which the crystalline behavior of PCL is affected by peptides in our composites. The first hypothesis arises from dispersing FF monomers into the PCL matrix, which potentially reduces the cross-linking efficiency in the polymer network. In this case, FF molecules could introduce a competition for $\mathrm{H}$-bonding between peptide end groups and oxygen sites along PCL chains, leading to lower chain-chain interactions. The second mechanism could be related to the interphase formed in the vicinities of FF-MNTs/PCL interfaces. In this scenario, higher availability of FF assemblies likely increases the volume of the interphase, exhibiting properties different from those observed in the bulk, and thus contributing to change in the average behavior of the FF-MNTs/PCL matrix.

\subsection{Mechanical properties and X-ray microtomography measurements}

Tensile tests were carried out for samples with formulations ranging from $2.5 \%$ to $50 \%$ (FF/PCL mass ratio). The results obtained from these assays are shown in Fig. 3A. The stressstrain curves contain information about strain and stress limits, Young's moduli and elongations at break. Elongation at break for samples containing $2.5 \%$ and $7.5 \%$ FF-MNTs are similar to pure PCL, whereas stress values are found to strongly increase even with the same elongation. For FF amounts higher than $7.5 \%$, stress at break increases from $2 \mathrm{MPa}$ to a steady value of $\sim 14.5 \mathrm{MPa}$. Similarly, elongation at break increases from $\sim 100 \%$ to $\sim 400 \%$, indicating that the composites become more flexible. We evaluate these values from Fig. 3B and the results are listed in Table S2 (ESI $\dagger)$. One observes that Young's modulus, maximum tensile strain and elongation at break are greatly improved, showing an overall enhancement of mechanical properties (mainly flexibility) upon addition of FF-MNTs to the PCL matrix. For instance, Young's modulus increased from 12.5 MPa, in bare PCL, to a maximum of 18.1 MPa in formulations with 50\% FF/PCL ratio (see Fig. 3C).

Dispersion, orientation and cohesion of the FF-MNTs within the matrix likely impact the mechanical properties of the composites. A net effect of different interactions at play during electrospinning of our polymer solutions - hydrogen bonds, $\pi$-stacking, hydrophobic interactions - improves the elastic properties upon increasing the amount of FF-MNTs in the sample. We hypothesize that this finding results from a better dissipation of the applied forces across the network. ${ }^{12,33}$ At lower amounts of FF-MNTs, elongation behaviour of the hybrid material is dominated by PCL characteristics - i.e., mechanical properties of the composite are closer to those found in peptide-free PCL. However, even at low FF concentrations, peptides and the

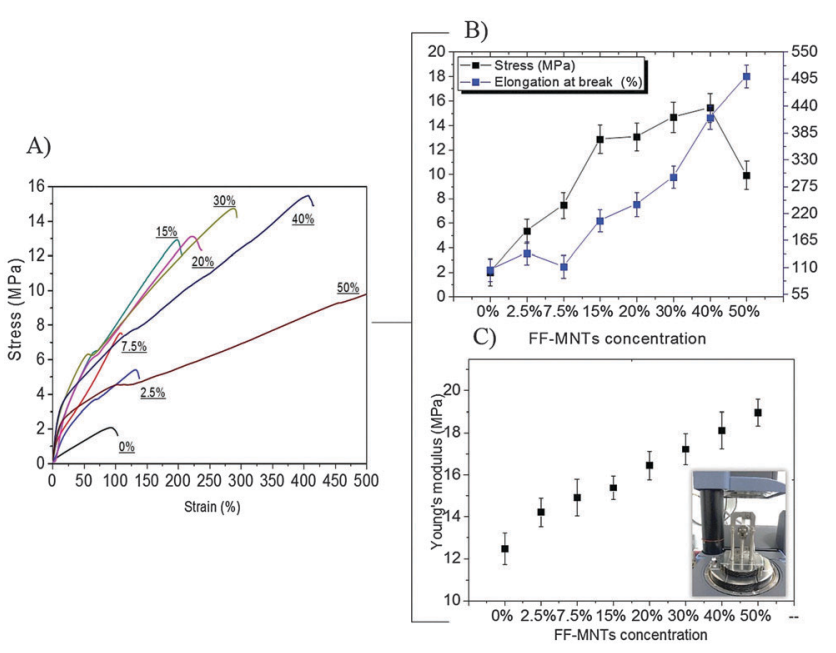

Fig. 3 (A) Stress-strain curves of electrospun samples; (B) stress and elongation at break behaviour against peptide concentrations in the formulations; (C) Young's modulus at different peptide concentrations. 
polymer interact to enhance stress at break, which could eventually be ascribed to the intercalation of FF monomers in the interstice of the polymer phase. Increasing the peptide concentration - and thus the amount of FF-MNTs in the composite - up to $40 \%$ makes FF-MNTs to coalesce, forming larger peptide-containing domains in the composite (as evidenced in the ESI $\dagger$, Fig. S2). These domains interact strongly with the amorphous region of the PCL, making it harder to break. The composite with 50\% FF-MNTs becomes very ductile to the extent that it is not possible to break the material in the strain range used in our experiments. In this case, mechanical properties of the higher concentration composites are strongly influenced by FF-MNTs exhibiting characteristics which are very distinct compared to bare PCL. ${ }^{33}$

Generally, matrices that are reinforced and oriented exhibit improved strength when tensile forces are applied parallel to the orientation axis. ${ }^{34}$ Here, despite the fact that membranes are apparently made up of randomly-distributed networks, a similar behaviour has been observed. We tentatively propose that this effect originates from a partially oriented system. In fact, although the electrospinning process leads to a random dispersion of the fibers onto the substrate (see microscope images), the applied electric field interacts with the dipole moment of FF molecules. In this case, such an interaction possibly induces a partial orientation of the polymeric fibers, which could exhibit some degree of local ordering and improve the mechanical properties as peptide concentration increases (see Fig. 4B).

$\mathrm{X}$-ray microtomography (Micro CT) yields insights into the internal structure of the scaffolds. It is a non-invasive technique using an incident X-ray beam at different scan angles, sectioning the object into 2D images, which are further combined for retrieving $3 \mathrm{D}$ reconstructions. Micro-CT is able to provide information about porosity and allows estimating empty space in the samples. Porosity measurements were performed on $\sim 500$ slices in volume for all concentrations. The $2 \mathrm{D}$ images show changes in the distribution of pores into polymeric matrices upon increasing the concentration of FF-MNTs.

Fig. 4A shows the cross-section micro-CT images of representative formulations (see ESI, $\dagger$ Fig. S4, for additional images of all formulations). The contrast effect observed in the images (yellow, white and blue colors) is based on different absorption of X-rays and how they reflect different composition domains in the samples. Vacancies (black color) decrease as the concentration of FF-MNTs increases, attesting that pores in the matrix are filled by the polymer.

Micro-CT data also shed light on the shape and alignment across the polymer network. Pure PCL fibres exhibited higher tendency to form globular clusters when compared to the composites. In contrast, addition of FF-MNTs resulted in higher alignment of the fibres, as schematically shown in Fig. 4B. In Fig. 4C, average porosity values obtained from micro-CT images are plotted as a function of the peptide amount in the matrix. In agreement with the behaviour exhibited by crystallinity degree, and the crystallite size derived from thermal and XRPD analyses, one observes that pore sizes also decay with an increasing amount
A)

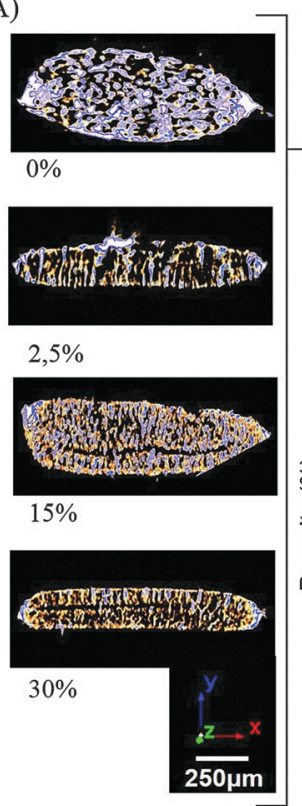

B)

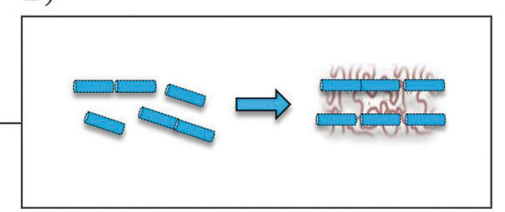

C)

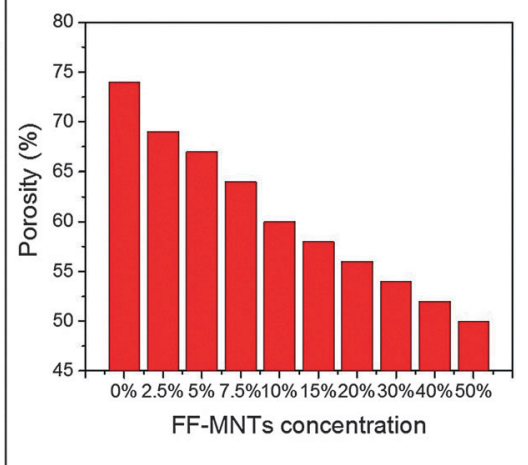

Fig. 4 Microtomography analyses of FF-MNTs/PCL samples. (A) Representative slices showing the inner morphology of the membranes, exposing the porous network; (B) schematic representation of the proposed organization framework where the presence of FF-MNTs induces local ordering on the polymer network reinforcing their mechanical properties. The blue tubes denote the PCL fibers. (C) Porosity of the membranes obtained from the distribution of pores for $\sim 500$ slices.

of peptide in the composite. These findings show that structural changes occurring at the supramolecular level are reflected on the microscopic structure of the samples and ultimately lead to enhanced elastic properties. ${ }^{34}$

\subsection{In vitro permeation studies}

In vitro permeation studies were performed for all composite formulations (with 10\%wt BZC) in comparison to a commercially available gel formulation (20\% BZC). Fig. 5A shows the behavior of the drug release curves from 30 minutes to 24 hours. The results of in vitro permeation profiles were plotted considering the permeation rate against time. The commercial gel formulation showed higher BZC permeated concentration compared to FF-MNTs/PCL scaffolds. Furthermore, the BZC permeation rate from films was reduced upon increasing the FF concentration (Fig. 5B); presumably, a consequence of the lower porosity observed in these complexes. After $24 \mathrm{~h}$, the BZC permeation rates from samples containing 7.5, 15, and 30\% $\mathrm{FF}$ were statistically different from that observed for the commercial gel 2.5\% FF $(p<0.05)$. These results suggest that the slow BZC permeation rate can be attributed to the possible interaction of the hydrophobic ester-type local anesthetic BZC with the polymeric networks (in particular hydrophobic polymers, such as PCL) in films. We observed similar results of release based on the FF-MNTs concentration. Samples containing higher concentrations promoted a slower release rate compared to the common gel. The release profiles were fitted by exponential functions to obtain the rate constant as a function of the peptide concentration. 

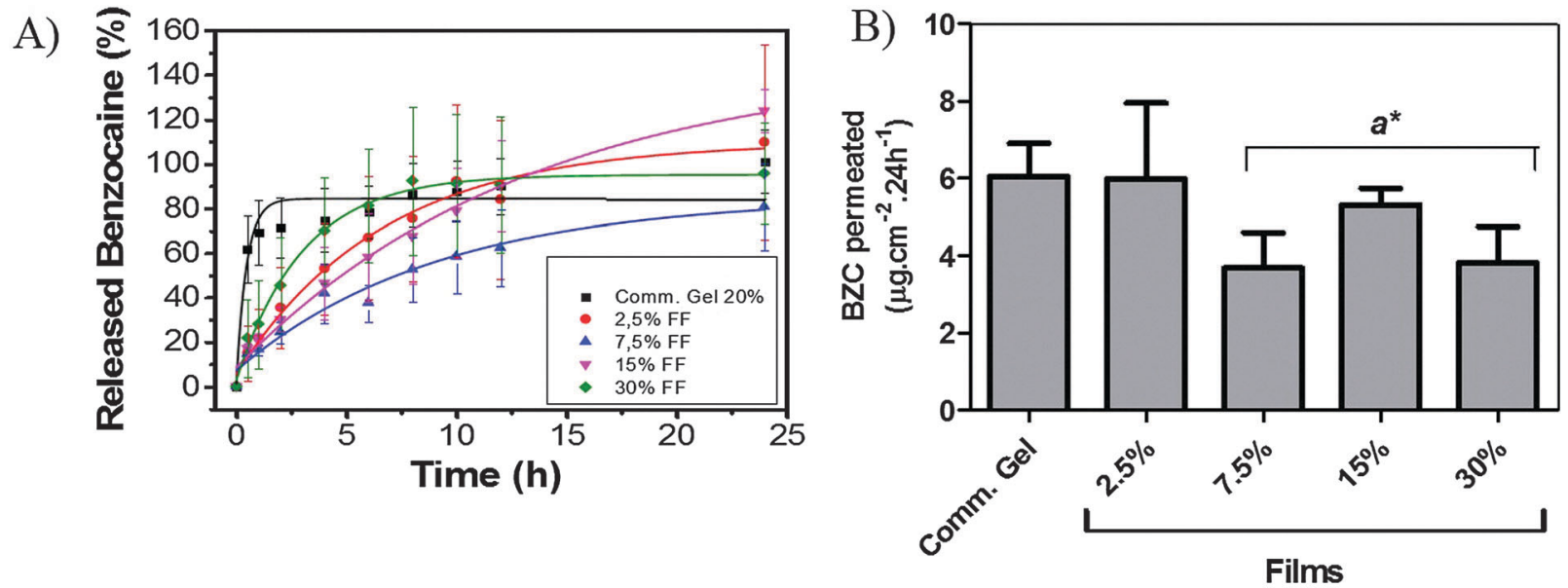

Fig. 5 (A) Release curves of BZC obtained by UV-Vis spectrophotometry. (B) BZC permeation rate at $24 \mathrm{~h}\left(a=\right.$ films versus commercial gel, $\left.{ }^{\star}=p<0.05\right)$.

The common gel presented a lag time of $\sim 0.5$ hours while it was gradually increased from 6.6 to 13.3 hours for FF concentrations from $2.5 \%$ to $15 \%$. The differences in the percentage concentration of BZC in the common gel $(20 \%)$ and the membrane $(10 \%)$ may explain the differences in the lag time values. Even for the membranes that have a lower BZC concentration, the $2.5 \%$ FF-MSNs films provided a similar permeation rate as the commercial gel, pointing to the possibility for the development of an effective delivery platform with lower drug concentrations (Fig. 5B).

BZC is a local anesthetic widely used for topical anesthesia, but its relatively slow absorption and/or fast biotransformation induces a short duration of action. Due to its application in a variety of dermatological products (gels, creams, ointments and sprays), new topical drug delivery systems have been developed. ${ }^{35,36}$ Thus, different materials such as synthetic polymers (carbomer polymers, poly-D,L-lactide-co-glycolide - PLGA, polyvinyl alcohol, PCL and peptide polymers) have attracted enormous attention. ${ }^{35-39}$

To the best of our knowledge, this is the first study where the addition of FF-MNTs to PCL has been shown to be an effective strategy for controlling the drug permeation rate with possible skin delivery applications. We note that increasing the FF concentration beyond a certain concentration reduces the release time. The optimum release time for the 15\% FF-MNTs concentration sample goes hand-in-hand with the mechanical properties of these fibers; the stress value for this concentration almost reaches saturation. These results suggest that the amount of $\mathrm{FF}$ in the matrix could be used as a variable to control the release rate from PCL/FF-MNTs hybrids.

\subsection{Biodegradability}

The biodegradation of PCL has been investigated against lipase and proteinase-K due to the high efficiency of these enzymes for hydrolysis of polymer chains. ${ }^{40}$ For this study, samples were incubated in solution with the enzymes for 25 hours. Fig. 6A shows the weight loss of pure PCL and PCL/FF-MNTs scaffolds for different concentrations of FF-MNTs. It is clearly observed that the weight loss is strongly impacted by the FF-MNTs concentration. Particularly, we have found that higher degradation rates are found when higher peptide amounts are present in the matrix.

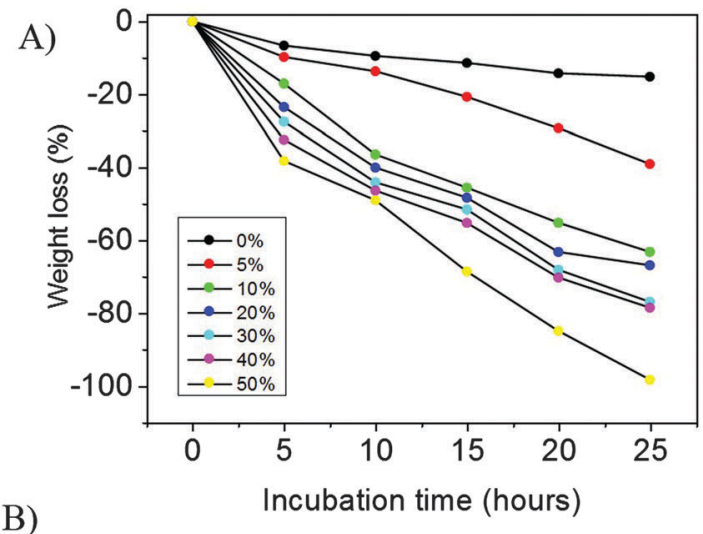

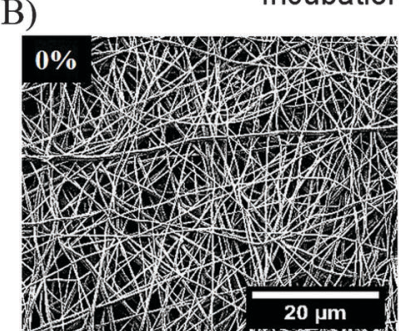

C)

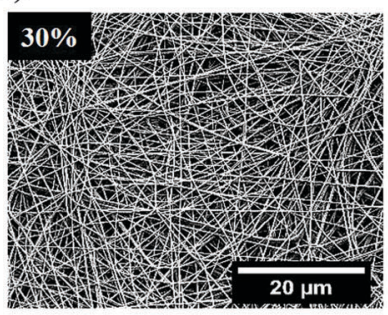

Before

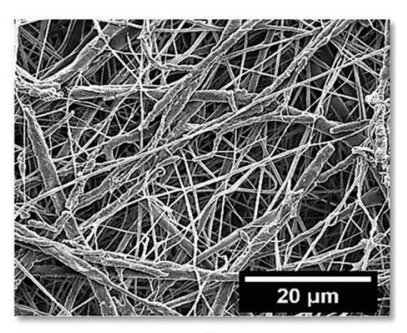

After

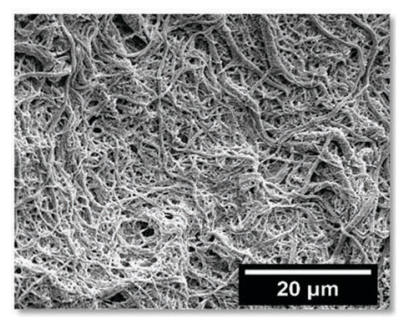

After
Fig. 6 (A) Percentage weight loss of PCL and PCL-FF-MNTs composites after 25 hours of incubation; (B and C) FE-SEM images of PCL and the $30 \%$ FF-MNTs sample before and after enzymatic incubation. The biodegradation occurs from the surface to the inside of the fiber. 
The greater weight loss with an increase in FF-MNTs concentration could be due to the decreased crystallinity and higher enzymatic affinity towards amide hydrolysis. ${ }^{28,41-44}$ Indeed, diminution of the fraction of ordered domains into the polymer matrix, associated with intercalation of peptide clusters in the interstice of the macromolecular scaffold, likely leads to hydrolysis across the membrane and favours destabilization of the whole polymer structure. ${ }^{41-44}$ SEM images obtained after a $25 \mathrm{~h}$ degradation period clearly show that increasing FF concentration leads to higher destruction of the fibers. Fig. 6B and $\mathrm{C}$ show, respectively, micrographs obtained before and after the degradation process from pure PCL and 30\% FF/PCL. The morphology of the fibers changes drastically for the $30 \%$ sample with a clear destruction of the fiber surface. The images of the samples after the biodegradation process for all concentrations are presented in Fig. S5 (ESI $\dagger$ ). Not only does the addition of FF-MNTs to PCL during the electrospinning process enhances mechanical strength and drug delivery rates, but also provides a tangible route towards green bio-chemistry.

\section{Conclusions}

We have presented a simple route for producing hybrid peptide/ polymer scaffolds built from non-covalent conjugation between FF and PCL. The strategy used in this work was based on electrospinning methods, where peptides and polymers are co-solubilized and subjected to strong electric fields in order to produce fibres with only a few hundred nanometer thicknesses. The structures arising from this procedure have been found suitable for producing homogenous networks and are capable of designing polymer membranes potentially usable as skin patches. We have observed that peptides are successfully incorporated into the polymer matrix and self-assemble into micro/nanotubes with remarkable mechanical properties. The elastic behaviour of the composite PCL fibres is strongly affected by peptides and both DSC and XRPD analyses have shown a close relationship between the mechanical properties and the crystalline structure. Specifically, we have found that peptides lead to a remarkable loss of crystallinity in the polymer, followed by a brittle-to-ductile transition at the macro-scale. Furthermore, micro-tomography assays have pointed out that average pore sizes in the fibers diminish upon increasing the peptide concentration. Unfortunately, the mechanisms as to why these structural changes occur remain somewhat elusive. However, we hypothesize that FF in the interstice of the polymer phase weakens inter-chain interactions and hinders cross-linking across the network, favoring the elastic behaviour of the hybrid material. Also, estimations on entropy changes introduced by mixing peptides with the polymer matrix show the growth of disorder and corroborate the breaking of crystalline ordering indicated by structural assays.

These membranes, exhibiting high flexibility, have been successfully used for modulating the release of benzocaine, an anesthetic widely used in pharmaceutics. It was demonstrated that, on scaffolds containing about $7.5-30 \% \mathrm{FF} / \mathrm{PCL}$, release rates are significantly slowed down compared to commercially available scaffolds. Interestingly, these same formulations also exhibit the highest elongation limits at break. Finally, we have shown that the scaffolds prepared through this simple strategy are easily degradable upon enzymatic attack; therefore, these biodegradable polymeric matrices have strong potential to behave as vehicles for sustained release of drugs directly into wound sites.

\section{Acknowledgements}

This work was supported by FAPESP (grant no. 2013/12997-0) and CNPq (grant no. 472197/2012-6). INCT in Bioanalytics (FAPESP grant no. 08/57805-2 and CNPq grant no. 573672/ 2008-3) is kindly acknowledged for grants. M. S. L., S. K. Jr. and E. R. S. acknowledge FAPESP for fellowships (Procs. no.: 2012/15481-1, 2012/01933-8 and 2013/12674-6). LNNano and LNLS staffs are kindly recognized for their invaluable help and providing access to SEM, XRPD and X-ray micro-CT facilities (proposals SEM - 18306, XRD1 - 16150, and Micro CT - 18458). M. S. L. thanks Dr Ligia Maria Manzine Costa for help on DMA and DSC assays.

\section{References}

1 G. Vilar, J. Tulla-Puche and F. Albericio, Curr. Drug Delivery, 2012, 9, 367-394.

2 D. E. Discher and A. Eisenberg, Science, 2002, 297, 967-973.

3 I. W. Hamley, Biomacromolecules, 2014, 15, 1543-1559.

4 T. A. Doll, S. Raman, R. Dey and P. Burkhard, J. R. Soc., Interface, 2013, 10, 20120740.

5 J. Pyun and K. Matyjaszewski, Chem. Mater., 2001, 13, 3436-3448. 6 V. L. Sedman, L. Adler-Abramovich, S. Allen, E. Gazit and S. J. B. Tendler, J. Am. Chem. Soc., 2006, 128, 6903-6908.

7 M. Reches and E. Gazit, Curr. Nanosci., 2006, 2, 105-111.

8 L. Adler-Abramovich, M. Reches, V. L. Sedman, S. Allen, S. J. B. Tendler and E. Gazit, Langmuir, 2006, 22, 1313-1320.

9 G. Zeng, L. Liu, D. Xia, Q. Li, Z. Xin, J. Wang, F. Besenbacher, T. Skrydstrup and M. Dong, RSC Adv., 2014, 4, 7516-7520.

10 S. Kogikoski Jr, C. P. Souza, M. S. Liberato, T. Andrade Filho, T. Prieto, F. F. Ferreira, A. R. Rocha, S. Guha and W. A. Alves, Phys. Chem. Chem. Phys., 2016, 18, 3223-3233.

11 K. Jayaraman, M. Kotaki, Y. Z. Zhang, X. M. Mo and S. Ramakrishna, J. Nanosci. Nanotechnol., 2004, 4, 52-65.

12 N. Even, L. Adler-Abramovich, L. Buzhansky, H. Dodiuk and E. Gazit, Small, 2011, 7, 1007-1011.

13 D. J. Rubin, H. T. Nia, T. Desire, P. Q. Nguyen, M. Gevelber, C. Ortiz and N. S. Joshi, Biomacromolecules, 2013, 14, 3370-3375.

14 L. W. Chow, A. Armgarth, J.-P. St-Pierre, S. Bertazzo, C. Gentilini, C. Aurisicchio, S. D. McCullen, J. A. M. Steele and M. M. Stevens, Adv. Healthcare Mater., 2014, 3, 1381-1386.

15 R. F. Silva, D. R. Araujo, E. R. Silva, R. A. Ando and W. A. Alves, Langmuir, 2013, 29, 10205-10212.

16 D. A. Bernards and T. A. Desai, Adv. Mater., 2010, 22, 2358-2362. 17 M. F. Canbolat, A. Celebioglu and T. Uyar, Colloids Surf., B, 2014, 115, 15-21. 
18 B. Wong, S.-H. Teoh and L. Kang, Drug Delivery Transl. Res., 2012, 2, 272-283.

19 T. K. Dash and V. B. Konkimalla, Mol. Pharmaceutics, 2012, 9, 2365-2379.

20 M. Chen, Y.-F. Li and F. Besenbacher, Adv. Healthcare Mater., 2014, 3, 1721-1732.

21 T. K. Dash and V. B. Konkimalla, J. Controlled Release, 2012, 158, 15-33.

22 S. Nicoli, P. Colombo and P. Santi, AAPS J., 2005, 7, E218-E223.

23 S. V. Fridrikh, J. H. Yu, M. P. Brenner and G. C. Rutledge, Phys. Rev. Lett., 2003, 90, 144502.

24 A. Mohamed, V. L. Finkenstadt, S. H. Gordon, G. Biresaw, D. E. Palmquist and P. Rayas-Duarte, J. Appl. Polym. Sci., 2008, 110, 3256-3266.

25 E. Chiellini and R. Solaro, Adv. Mater., 1996, 8, 305-313.

26 J. Albuerne, L. Marquez, A. J. Muller, J. M. Raquez, P. Degee, P. Dubois, V. Castelletto and I. W. Hamley, Macromolecules, 2003, 36, 1633-1644.

27 H. Kweon, M. K. Yoo, I. K. Park, T. H. Kim, H. C. Lee, H.-S. Lee, J.-S. Oh, T. Akaike and C.-S. Cho, Biomaterials, 2003, 24, 801-808.

28 J. Ryu and C. B. Park, Biotechnol. Bioeng., 2010, 105, 221-230.

29 M. I. Souza, E. R. Silva, Y. M. Jaques, F. F. Ferreira, E. E. Fileti and W. A. Alves, J. Pept. Sci., 2014, 20, 554-562.

30 N. Amdursky, P. Beker, I. Koren, B. Bank-Srour, E. Mishina, S. Semin, T. Rasing, Y. Rosenberg, Z. Barkay, E. Gazit and G. Rosenman, Biomacromolecules, 2011, 12, 1349-1354.

31 H. Bittiger, R. Marchess and W. D. Niegisch, Acta Crystallogr., Sect. B: Struct. Sci., 1970, B26, 1923-1927.
32 L. E. Alexander, X-ray diffraction methods in polymer science, Wiley-Interscience, New York, 1969.

33 A. W. Martinez, J. M. Caves, S. Ravi, W. Li and E. L. Chaikof, Acta Biomater., 2014, 10, 26-33.

34 V. Thomas, M. V. Jose, S. Chowdhury, J. F. Sullivan, D. R. Dean and Y. K. Vohra, J. Biomater. Sci., Polym. Ed., 2006, 17, 969-984.

35 D. R. Araujo, C. Padula, C. M. Saia Cereda, G. R. Tofoli, R. B. Brito, Jr., E. de Paula, S. Nicoli and P. Santi, Pharm. Res., 2010, 27, 1677-1686.

36 D. R. Araujo, D. C. Silva, R. M. Barbosa, M. Franz-Montan, C. M. S. Cereda, C. Padula, P. Santi and E. Paula, Expert Opin. Drug Delivery, 2013, 10, 1551-1563.

37 B. Jeong, S. W. Kim and Y. H. Bae, Adv. Drug Delivery Rev., 2002, 54, 37-51.

38 C. Padula, A. Fulgoni and P. Santi, Skin Research and Technology, 2010, 16, 125-130.

39 C. Padula, C. Chiapponi, M. T. Dibari, A. Deriu, F. Sonvico, S. Barbieri, J. Ollivier and P. Santi, J. Pharm. Sci., 2010, 99, 4277-4284.

$40 \mathrm{H}$. Tsuji, Y. Kidokoro and M. Mochizuki, Macromol. Mater. Eng., 2006, 291, 1245-1254.

41 S. Khanra, T. Cipriano, T. Lam, T. A. White, E. E. Fileti, W. A. Alves and S. Guha, Adv. Mater. Interfaces, 2015, 2, 1500265.

42 M. J. Jenkins and K. L. Harrison, Polym. Adv. Technol., 2008, 19, 1901-1906.

43 H. Cai, V. Dave, R. A. Gross and S. P. McCarthy, J. Polym. Sci., Part B: Polym. Phys., 1996, 34, 2701-2708.

44 M. Reches and E. Gazit, Science, 2003, 300, 625-627. 